\title{
Anmerkungen zu Otto Blumenthals Vortrag ,„̈̈ber das mathematische Bildungswesen im heutigen Rußland“" (Aachen 1932)
}

\section{Volkmar Felsch}

Online publiziert: 9. Januar 2017

(C) Springer-Verlag Berlin Heidelberg 2016

Der Aachener Mathematikprofessor Otto Blumenthal ${ }^{1}$ hielt den vorstehend abgedruckten Vortrag am 30. März 1932 auf der 34. Hauptversammlung des Vereins zur Förderung des mathematischen und naturwissenschaftlichen Unterrichts in Aachen. ${ }^{2}$ Dieser bisher unveröffentlichte Text ist in doppelter Hinsicht bemerkenswert, zum einen wegen seines mathematik-didaktischen Inhalts, zum anderen aber auch wegen der Rolle, die er in dem Verfahren spielte, das ein Jahr später zu Blumenthals Entlassung aus dem Lehramt führte. ${ }^{3}$

Am 18. März 1933 schickte der nationalsozialistisch gesteuerte AStA der TH Aachen einen Denunziationsbrief an den Wissenschafts-Minister in Berlin, in dem heftige Vorwürfe gegen einige Professoren der TH erhoben wurden. Einer der davon Betroffenen war Blumenthal, über den es in dem Brief hieß: ${ }^{4}$

\footnotetext{
1 Vor der Berufung auf seinen Lehrstuhl an der TH Aachen gehörte Otto Blumenthal in Göttingen zum engeren Kreis der Schüler von David Hilbert. Eine Dokumentation seiner Göttinger Zeit findet sich in dem demnächst erscheinenden Buch: Otto Blumenthal, Ausgewählte Briefe und Schriften. Band I, 1897-1922. Herausgegeben von David E. Rowe, Springer, Heidelberg, 2017.

2 Das Originalmanuskript des Vortrags befindet sich in Duisburg im Landesarchiv NRW Abteilung Rheinland in der Akte Regierung Aachen, 20065.

3 Eine ausführlichere Darstellung der im Folgenden zusammengefassten Ereignisse findet sich in Volkmar Felsch: Otto Blumenthals Tagebücher. Ein Aachener Mathematikprofessor erleidet die NS-Diktatur in Deutschland, den Niederlanden und Theresienstadt. Hartung-Gorre Verlag, Konstanz, 2011, S. 31-43.

4 Der hier zitierte Brief befindet sich im Hochschularchiv der RWTH Aachen. Mit Ausnahme des Soziologen Alfred Meusel, der später als Historiker in der DDR wirkte, waren von den in diesem und einem nachfolgenden Brief des AStA an den Minister erhobenen Anschuldigungen ausschließlich jüdische Mitglieder des Lehrkörpers der Hochschule betroffen.
}

V. Felsch $(\bowtie)$

Aachen, Deutschland

E-Mail: volkmar.felsch@gmx.de 
Es kann deutsch gesinnten Studenten nicht mehr länger zugemutet werden, sich von Freunden des russischen Bolschewismus unterrichten und prüfen zu lassen. Wie weitreichend der Einfluss sein kann, den ein marxistisch eingestellter und sich als Freund der Sowjetunion bekennender Professor auf die ihm durch den Lehrplan zugewiesenen Studierenden auszuüben in der Lage und gewillt ist, zeigt sich am Beispiele des ordentlichen Professors Dr. Blumenthal, der [...] schon lange den Unwillen unserer national gesinnten Studierenden erregt [...]. Durch seine Vorträge, die Professor Blumenthal - zum Teile auch in der Hochschule selbst - über seine Erlebnisse auf einer Russlandsreise vor einiger Zeit gehalten hat und durch die er mit beitrug zu einem gewissen „Sowjetkult“, ist es ihm gelungen, eine grössere Zahl von Lehramtskandidaten, die dereinst die Erzieher der deutschen Jugend sein sollen, für die Gesellschaft der Freunde des neuen Russlands zu werben und sie für eine aktive Tätigkeit in dieser Gesellschaft einzuspannen. Diese Lehramtskandidaten und-kandidatinnen unterstützten auch in der Hauptsache durch ihre Unterschriften die antifaschistische Wahlliste bei den letzten Studentenschaftswahlen. Wir bezweifeln allerdings, dass diese Studierenden, die Herrn Professor Blumenthal zur mathematischen Ausbildung zugewiesen sind, sämtlich aus innerer Überzeugung „Antifaschisten" sind, glauben vielmehr, dass das Gefühl, von ihm in den Prüfungen abhängig zu sein, sie zur Unterstützung der Antifaschistenliste bewogen haben könnte. Wir müssen gerade diesen Verdacht besonders unterstreichen, um verständlich zu machen, dass unsere Studierenden nicht länger mehr gewillt sind, sich von marxistisch und sowjetistisch eingestellten Professoren prüfen zu lassen.

In Berlin wurden diese Unterstellungen für so schwerwiegend gehalten, dass das Ministerium die TH Aachen am 31. März telegrafisch anwies, ,bis zur Entscheidung über die AStA-Eingabe von der Beteiligung der in dem Schreiben genannten Professoren an den Prüfungen abzusehen". 5 Der Rektor informierte Blumenthal über die gegen ihn erhobenen Vorwürfe und bat ihn um eine schriftliche Stellungnahme, die Blumenthal auch noch am selben Tage ablieferte. Darin schrieb er:

1.) Ich habe niemals direkt oder indirekt irgendwelche marxistische oder sowjetistische Propaganda getrieben. Dies ist schon allein deshalb unmöglich, weil ich kein Anhänger dieser Lehren bin oder jemals war.

2.) Meine Angehörigkeit zu der „Gesellschaft der Freunde des neuen Russlands “ erklärt sich dadurch, dass ich seit dem Jahre 1900 aus Interesse an der russischen schöngeistigen und wissenschaftlichen Literatur mich mit russischer Sprache beschäftige, es darin zu einer gewissen Fertigkeit gebracht habe und auch schon im Jahre 1900 in Russland gereist bin. Die „Gesellschaft“ stellt sich lediglich das Studium der Verhältnisse im jetzigen Russland zur Aufgabe, objektiv und ohne jede Parteinahme. Der Name ist irreführend. [...] Ob an

\footnotetext{
5 Zitiert aus einem Brief des Rektors an das Ministerium vom 1. April 1933. Dieser Brief und auch alle im Folgenden zitierten Briefe befinden sich in Duisburg im Landesarchiv NRW Abteilung Rheinland (in den Akten 20065 Regierung Aachen und NW5-831 Kultusministerium).
} 
der Hochschule studierende Lehramtskandidaten der „Gesellschaft“ angehört haben, weiss ich nicht, jedenfalls habe ich niemals jemand dazu veranlasst.

3.) Nachdem ich bereits im Jahre 1930 auf Einladung mathematischer Kollegen, die von meiner Kenntnis der russischen Sprache wussten, an einem Mathematiker-Kongress in Charkow teilgenommen hatte, habe ich 1931 eine zweite Reise nach Russland unternommen, unter anderem zum Zwecke des Studiums der dortigen Unterrichtsverhältnisse an Universitäten und Technischen Hochschulen. Ich habe dabei den Eindruck gewonnen, dass die dortige Art des Seminarbetriebs, der sich eng an die Lektüre eines Lehrbuchs anschliesst, gegenüber der in Aachen bis dahin üblichen Methode der Vortragsseminare Vorteile hat. Dies habe ich mehrmals in Seminarbesprechungen erwähnt und habe auch in dem letzten Winter ein Seminar mit gemeinsamer Lektüre abgehalten. Dies ist der einzige Zusammenhang, in dem ich Studenten gegenüber auf Sowjetrussland hingewiesen habe. Aus dieser Aeusserung auf Sowjetfreundlichkeit zu schließen, ist gänzlich abwegig, da es sich allein um eine Frage der Lehrtaktik handelt.

4.) Ich habe über meine russischen Reisen in zwei Vorträgen berichtet, die ich Ihnen beide im Original einreiche. [...]

5.) Ich würde es als eine schwere Verleumdung empfinden, wenn man mir den Vorwurf machen wollte, dass ich mich bei Prüfungen irgendwie durch die politische oder gesellschaftliche Einstellung meiner Prüflinge beeinflussen lasse. [Es folgt eine längere Argumentation zur Entkräftigung dieses Vorwurfs.]

Der Rektor schickte diese Stellungnahme sofort nach Berlin weiter, aber auch Blumenthals Widersacher blieben nicht untätig. In zwei weiteren Briefen an den Minister bekräftigten der Aachener Kreisverband des Nationalsozialistischen Lehrerbundes (am 5. April) und der AStA (am 10. April) die in dem ersten AStA-Brief erhobenen Anschuldigungen und forderten energisch die Entfernung von Blumenthal und vier weiteren Professoren aus dem Lehramt. ${ }^{6}$

Der zuständige Ministerialdirektor im Ministerium, Georg Gerullis, leitete daraufhin ein Ermittlungsverfahren gegen diese fünf Professoren ein. Am 19. April schrieb er an den AStA:

Nachdem die Bestimmungen des Gesetzes über die Wiederherstellung des Berufsbeamtentums bekannt gemacht sind, ersuche ich an der Hand dieser Bestimmungen anzugeben, welche Gründe für die Entfernung der Professoren Blumenthal, Meusel und Fuchs und etwaiger anderer aus ihren Lehrämtern vorgebracht werden. Die Gründe müssen durch Beweise belegt werden. Der Leiter der Hochschulabteilung wird gegebenenfalls an Ort und Stelle die Angelegenheit prüfen und die Ankläger den in Frage kommenden Professoren gegenüberstellen. Die Äußerung ersuche ich umgehend einzureichen.

Der AStA und der Lehrerbund kamen dieser Aufforderung nicht nach. Trotz mehrfacher Nachfrage zeigten sie sich außerstande, ihre Vorwürfe zu konkretisieren oder irgendwelche Beweise vorzulegen. Eine Gegenüberstellung, wie sie auch von

\footnotetext{
${ }^{6}$ Es handelte sich um die Professoren Walter Fuchs, Ludwig Hopf, Carl-Max Maedge und Alfred Meusel.
} 
Blumenthal gefordert wurde, lehnten sie ab. Stattdessen schufen sie am 27. April 1933 eigenmächtig neue Fakten: Studentische Hilfspolizisten verhafteten die Professoren Blumenthal und Meusel und übergaben sie der politischen Polizei, die sie daraufhin in sogenannte ,Schutzhaft“ nahm.

Nach 15 Tagen Haft wurde Blumenthal am 11. Mai zwar wieder auf freien Fuß gesetzt, aber zu seiner großen Enttäuschung wurde seine Suspendierung nicht aufgehoben. Stattdessen beauftragte das Ministerium am 16. Mai den Aachener Regierungspräsidenten in seiner Eigenschaft als Staatskommissar für die Aachener Hochschule, die Ermittlungen zu den Vorwürfen gegen ihn und die vier anderen Professoren durchzuführen.

Die Ermittlungen gingen schleppend voran. Erst am 13. Juli wurde Blumenthal zu einer halbstündigen Vernehmung im Regierungspräsidium vorgeladen, an deren Ende er ein Protokoll seiner Aussagen unterschrieb, in denen er unter anderem gesagt hatte:

Ich bestreite irgendwie marxistisch oder gar sowjetistisch gesinnt zu sein oder mich betätigt zu haben. Insbesondere ist es nicht wahr, dass ich in irgend einer Form für die Sowjetrepublik und ihre staatspolitischen Einrichtungen Propaganda getrieben habe. Ich habe mich in meinen Vorträgen, die bereits in Berlin vorgelegt sind, bemüht, sachlich und kritisch zu sein. Ich habe keinesfalls eine Verherrlichung getrieben. Insbesondere habe ich mich gegen den Geist der Unduldsamkeit, der das sowjetistische Prinzip beherrscht, in jeder Form gewandt. Meine Vorlesungen beziehen sich auf Mathematik und haben das sachliche Gebiet nie verlassen. Ich habe die Sowjetunion nur in meinen Seminarvorbesprechungen erwähnt, als es sich darum handelte, eine bestimmte didaktische Methode, die ich dort kennen gelernt hatte, probeweise auch in unseren Seminarübungen zu verwenden.

Vier Tage später, am 17. Juli 1933, wurden die Ermittlungen im Aachener Regierungspräsidium abgeschlossen und ein Bericht nach Berlin geschickt. Er beschäftigte sich zunächst mit den Professoren Fuchs und Meusel, bevor er auf Blumenthal, Hopf und Maedge zu sprechen kam:

Die drei anderen Professoren bestreiten entschieden, irgendwie sich kommunistisch oder marxistisch betätigt zu haben. Sie legen Verwahrung dagegen ein, dass sie auch nur so gesinnt seien. Besonderes Tatsachenmaterial: Zeugenaussagen, Auszüge aus den Vorlesungen oder ähnliches konnte trotz aller Aufforderungen von keiner Seite beigebracht werden.

Besondere Vorwürfe richten sich gegen Prof. Blumenthal und seine Vorträge über seine Reisen durch Russland. Um diesen Vorwurf zu entkräften, hat Blumenthal die Abschriften der beiden Vorgänge der dortigen Stelle vorgelegt. Die Abschrift des einen Vortrags befindet sich bei den Akten.

Aus diesen Vorträgen dürfte sich wohl nicht eine kommunistische Denkweise oder gar eine Verherrlichung der kulturellen Entwicklung der Sowjetrepublik herauslesen lassen. Blumenthal gilt allgemein [...] als Idealist. Ich glaube auch, dass er mehr aus Lehrgründen als aus irgend einem anderen Grunde sich mit 
der Sowjetrepublik beschäftigt hat. Allerdings bestand dann die Gefahr, wie es geschehen ist, dass dieses Streben missverstanden werden konnte.

Obwohl damit die durch den AStA und den Lehrerbund gegen Blumenthal erhobenen Vorwürfe gegenstandslos geworden waren, blieb er auch weiterhin beurlaubt. Wie alle Beamten musste er den ,Fragebogen zur Durchführung des Gesetzes zur Wiederherstellung des Berufsbeamtentums vom 7. April 1933“ ausfüllen, und eine Frage darin wurde ihm zu Verhängnis. Am 29. September 1933 wurde er endgültig aus dem Staatsdienst entlassen. Der Entlassungsgrund lautete: „Mitglied der Liga für Menschenrechte seit 1919/21“".

Bemerkenswert ist, dass in dem 1938 erschienenen Bericht von Wilhelm Lorey über die 34. Hauptversammlung des Vereins zur Förderung des mathematischen und naturwissenschaftlichen Unterrichts Blumenthal und sein Vortrag überhaupt nicht erwähnt werden, ${ }^{8}$ obwohl er sogar, zusammen mit dem Rektor der TH und dem Oberbürgermeister der Stadt Aachen, Mitglied des fünfköpfigen Ehren-Ausschusses dieser Versammlung gewesen war.

Im Juli 1939 emigrierte Otto Blumenthal mit seiner Frau in die Niederlande, wo sie 1940 von den deutschen Besatzungstruppen eingeholt wurden. Nach langen Schikanen starb Mali Blumenthal im Mai 1943 im niederländischen Lager Westerbork, Otto Blumenthal starb im November 1944 in Theresienstadt.

\footnotetext{
7 Siehe: Sybille Gerstengarbe: Die erste Entlassungswelle von Hochschullehrern deutscher Hochschulen aufgrund des Gesetzes zur Wiederherstellung des Berufsbeamtentums vom 7.4.1933. Berichte zur Wissenschaftsgeschichte 17 (1994), S. 17-39, hier S. 34.

${ }^{8}$ Siehe: Der Deutsche Verein zur Förderung des mathematischen und naturwissenschaftlichen Unterrichts. E. V. 1891-1938. Ein Rückblick zugleich auch auf die mathematische und naturwissenschaftliche Erziehung und Bildung in den letzten fünfzig Jahren. Verfaßt im Auftrag des letzten Vorstands von Wilhelm Lorey. Verlag Otto Salle, Frankfurt am Main, 1938, S.101-103.
} 